MATEC Web of Conferences 11,01035 (2014)

DOI: $10.1051 /$ matecconf $/ 20141101035$

(C) Owned by the authors, published by EDP Sciences, 2014

\title{
Comportement en flexion des bétons fibrés sous chargement cyclique
}

\author{
${ }^{1}$ Bensaid Boulekbache, ${ }^{1}$ Mostefa Hamrat, ${ }^{2}$ Mohamed Chemrouk and ${ }^{3}$ Sofiane Amziane \\ ${ }^{1}$ Département de Génie Civil, Université Hassiba Benbouali, Chlef, Algérie \\ ${ }^{2}$ Université des Sciences et Technologie Houari Boumediene, Alger, Algérie \\ ${ }^{3}$ Polytech'Clermont, Université Blaise Pascal, Clermont Ferrand, France
}

\begin{abstract}
Ce papier présente les résultats d'une étude expérimentale sur le comportement en flexion des bétons de fibres métalliques. On étudie l'effet de la rhéologie du béton sur l'orientation des fibres et l'influence de l'orientation sur les propriétés mécaniques. La rigidité de l'ancrage des fibres étudiée par les essais cycliques est liée aux caractéristiques rhéologiques et mécaniques de la matrice. Les résultats montrent que la fluidité des bétons est un paramètre essentiel de l'orientation des fibres. Dès lors que l'on obtient une orientation dans le sens de l'efficacité mécanique, la résistance à la flexion est nettement améliorée.
\end{abstract}

\section{Introduction}

Le béton de fibres est un matériau qui a connu ces derniers temps beaucoup de développements et nombreuses sont les études qui ont été effectuées durant les trois dernières décennies. L'ajout des fibres métalliques a un effet positif sur les caractéristiques mécaniques des bétons. Dans ce domaine, on peut citer l'amélioration simultanée des propriétés mécaniques et de la ductilité. Par ailleurs, la formation et la propagation des fissures sont retardées et les tailles de ces fissures sont largement réduites [1]. L'idée de renforcer les bétons avec des fibres métalliques paraît un bon moyen pour leur conférer une meilleure ductilité.

La flexion est le type de sollicitation le plus rencontré dans les structures. Bien qu'il présente un effet d'échelle dû au gradient d'effort sur la hauteur, il est l'essai le plus utilisé au laboratoire grâce à sa simplicité et reste un bon moyen de comparaison entre les différents bétons de fibres. La rhéologie du matériau et l'effet de paroi généré par la géométrie du coffrage, sont ceux auxquels l'orientation des fibres est la plus sensible [2].

\section{Programme expérimental}

\subsection{Matériaux et matériel utilisés}

Trois types de bétons sont formulés selon les proportions indiquées dans le tableau 1 . Les fibres utilisées sont des fibres métalliques munies de crochets à leurs extrémités de résistance à la traction de $1100 \mathrm{MPa}$. On a utilisé trois dosages volumiques en fibres $0 \%, 0,5 \%$ et $1 \%$ et deux élancements 65 et 80 .
Les bétons sont confectionnés dans un malaxeur d'une capacité de 100 litres. Des prismes de dimensions $15 \times 15 \times 70 \mathrm{~cm}$ sont testés en flexion à quatre points. Une entaille de $1 \mathrm{~cm}$ de profondeur est effectuée au milieu des prismes pour favoriser la localisation de la fissure dans la partie étudiée. Les éprouvettes destinées aux essais de compression sont des éprouvettes cylindriques $11 \times 22 \mathrm{~cm}$. Elles sont soumises à une vibration externe (table vibrante) sauf en ce qui concerne le béton auto-plaçant. Les éprouvettes sont conservées dans une salle dotée d'une climatisation qui assure une température de $20{ }^{\circ} \mathrm{C}$ et une humidité relative de $90 \pm 5 \%$. Trois éprouvettes pour chaque essai ont été testées. Les résultats des essais des bétons à l'état frais sont présentés dans le tableau 1 .

Les tests de flexion sont effectués sur une machine " Zwick Roller 4 colonnes" de capacité $250 \mathrm{kN}$ (figure 1). En cours d'essai, on effectue plusieurs décharges aux flèches $0.2,0.5,1,2$ et $4 \mathrm{~mm}$ de façon à observer l'évolution de l'état d'ancrage des fibres et évaluer l'apport des fibres sur le comportement post-fissuration. Une caméra numérique $3 \mathrm{D}$ a été utilisée sur la zone de flexion, permettant la détection de la première fissure et l'évolution du processus de fissuration (figure 1).

Les paramètres étudiés sont: résistance du béton, dosage et élancements des fibres. L'analyse porte également sur l'orientation des fibres et leur ancrage au cours des essais cycliques.

- BO : Béton Ordinaire, BAP: Béton Auto-Plaçant , BHP : Béton à Hautes Performances

- BOF x-y, BAPF x-y, BHPF x-y

La lettre " $F$ " désigne béton fibré,

$\mathrm{x}$ : longueur des fibres, $\mathrm{y}$ : dosage des fibres. 
Tableau 1. Formulation et caractéristiques rhéologiques des bétons testés

\begin{tabular}{|l|c|c|c|c|c|c|}
\hline Composition $\left(\mathrm{kg} / \mathrm{m}^{3}\right)$ & BO & BOF & BAP & BAPF & BHP & BHPF \\
\hline Ciment & 275 & 275 & 425 & 425 & 425 & 425 \\
\hline Fumée de silice & 0 & 0 & 0 & 0 & 42,5 & 42,5 \\
\hline Filler calcaire & 90 & 90 & 200 & 200 & 90 & 90 \\
\hline Eau $\left(\mathrm{kg} / \mathrm{m}^{3}\right)$ & 178 & 178 & 192 & 192 & 161 & 161 \\
\hline Superplastifiant & 0 & 1,95 & 5,10 & 7,22 & 4,25 & 6,75 \\
\hline Gravier 4/10 & 910 & 900 & 825 & 814 & 825 & 814 \\
\hline Sable 0/4 & 830 & 820 & 750 & 740 & 750 & 740 \\
\hline E/L & 0,49 & 0,49 & 0,31 & 0,31 & 0,29 & 0,29 \\
\hline Affaissement $(\mathrm{cm})$ & 24 & 21 & $/$ & $/$ & 16 & 10 \\
\hline Etalement $(\mathrm{cm})$ & $/$ & $/$ & 70 & 65 & $/$ & $/$ \\
\hline Contrainte seuil $(\mathrm{Pa})$ & 45 & $/$ & 36 & $/$ & 120 & $/$ \\
\hline
\end{tabular}

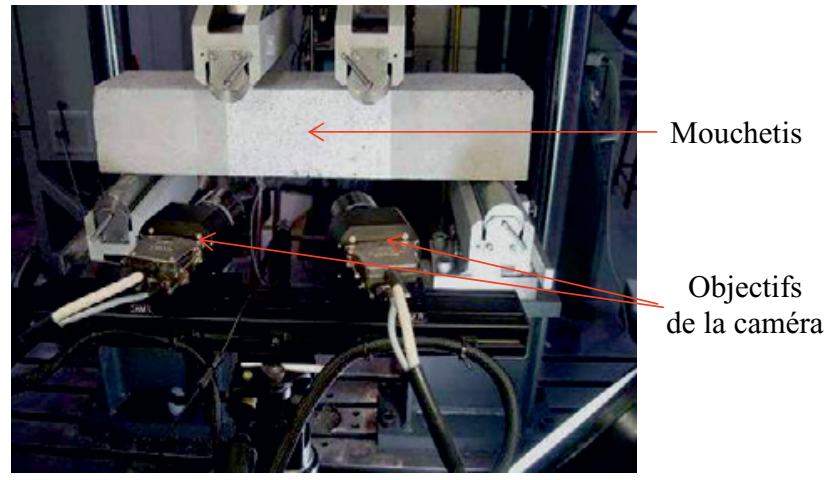

Fig. 1. Dispositif expérimental de l'essai de flexion

\section{Résultats et discussions}

\subsection{Orientation des fibres}

L'orientation des fibres des différentes configurations a été étudiée. La répartition des fibres dans la matrice peut être modifiée selon la rhéologie du béton. Nous avons compté les fibres dans la section de prismes rompues par flexion. Le nombre de fibres dépend du dosage volumique $V_{f}$, des dimensions des fibres et de leur état de distribution. Il est exprimé par l'expression suivante [3] :

$$
N=\alpha \frac{V_{f}}{A_{f}} A
$$

Avec $\alpha$ : coefficient d'orientation,

$A$ : section de l'éprouvette; $A_{f}$ : section de la fibre.

La figure 2 montre la moyenne des coefficients d'orientation. Nous constatons que les fibres avec un élancement de 80 sont mieux orientées que celles avec un élancement de 65. L'effet de la vitesse d'écoulement sur la rotation des fibres devient important avec la diminution de l'allongement et donc les fibres s'orientent aléatoirement [4]. En outre, pour des dosages de 0,5\%, on a eu de bonnes orientations par rapport aux dosages de $1 \%$, cette différence est due à la rhéologie des bétons affectée par le surplus de fibres qui ont un effet néfaste sur la mise en œuvre des bétons.

Il est important de noter que les bétons fluides (BOF et BAPF) ont eu des facteurs d'orientation les plus élevés et une distribution plus homogène sur toutes les poutres.

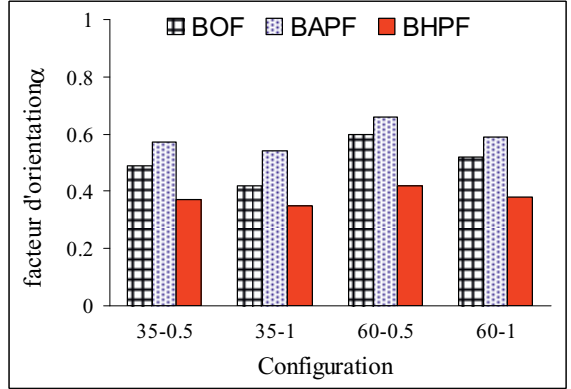

Fig. 2. Facteurs d'orientation des différentes configurations

\subsection{Résistances mécaniques}

Les bétons testés se différencient par leur résistance à la compression à 28 jours 29,61 et $80 \mathrm{MPa}$ pour le béton ordinaire, autoplaçant et à hautes performances respectivement et par la fluidité (BAP très fluide, BO fluide et BHP plastique).

Les charges de $1^{\text {ère }}$ fissuration et ultimes sont présentées dans la figure 3 . Les charges de $1^{\text {ère }}$ fissuration sont influencées par la résistance de la matrice (figure 3(a)), par contre les charges ultimes dépendent du dosage et de l'élancement des fibres (figure 3(b)). L'amélioration de la résistance à la première fissuration est due au transfert de l'effort à partir de la matrice aux fibres par adhérence. L'effort est ainsi partagé par les fibres et la matrice.

Une formule de régression pour prédire la résistance à la flexion $f_{u}$ d'un béton de fibres en fonction du béton sans fibres $f_{0}$ et le facteur de fibres $V_{\mathrm{f}}(\mathrm{L} / \mathrm{d})$ est donnée comme suit :

$f_{u}=f_{0}+3,04 V_{f}(L / d)$

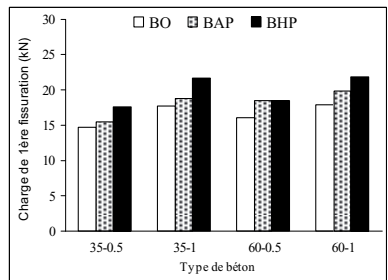

(a) $1^{\text {ère }}$ fissuration

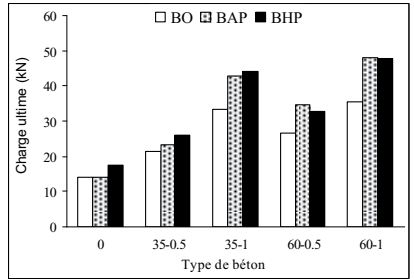

(b) ultimes
Fig. 3. Charges de $1^{\text {ère }}$ fissuration et ultimes

La figure 4 montre les courbes enveloppes charge-flèche déduites des courbes cycliques. On constate que le matériau avec $1 \%$ de fibres présente un comportement mécanique très amélioré par rapport au matériau avec $0,5 \%$ surtout au niveau du pic. A noter que dans la matrice de haute résistance (BHP), une baisse soudaine de la charge s'est produite juste après la charge maximale. Pour les BAPF et après une redistribution de contraintes, plusieurs fissures apparaissent après la première fissure, signe d'une bonne distribution et orientation des fibres dans la poutre. 


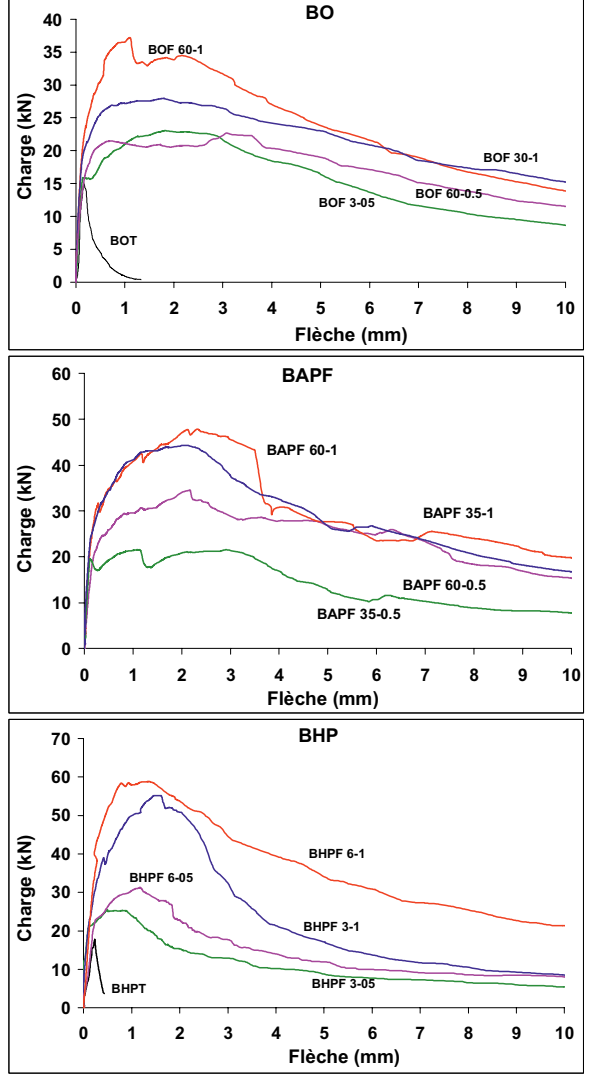

Fig. 4. Courbes charge-flèche des bétons testés

L'effet de la résistance du béton sur le comportement en flexion n'est pas important, sauf pour un dosage de $1 \%$, l'effet est plus ou moins remarquable, dû à l'augmentation des frottements entre la matrice et les fibres qui sont plus nombreuses dans ce cas.

Pour une variation de dosage de $0,5 \%$ à $1 \%$ de fibres d'élancement 65 , la reprise de charge passe de $147 \%$ à $189 \%$ pour les BOF, de $150 \%$ à $228 \%$ pour les BAPF et de $147 \%$ à $203 \%$ pour les BHPF. Pour l'élancement 80 avec un dosage variant de $0,5 \%$ à $1 \%$, la reprise passe de $166 \%$ à $198 \%$ pour les $\mathrm{BOF}$, de $187 \%$ à $242 \%$ pour les BAPF et de $178 \%$ à $218 \%$ pour les BHPF.

L'élancement n'a pas un effet significatif en comparaison avec le dosage. Néanmoins, nous constatons que les bétons avec des fibres d'élancement 80 ont un comportement mécanique en flexion meilleure que celui relatif à l'élancement 65 . L'hypothèse la plus plausible pour expliquer cette différence est que la surface frottante totale entre les fibres et la matrice pour l'élancement 80 est supérieure à celle relative aux fibres d'élancement 65 malgré que les fibres d'élancement 65 sont plus nombreuses dans la matrice que les fibres d'élancement 80 pour un même dosage volumique.

En général, les bétons autoplaçants ont prouvé une meilleure performance dans la reprise de charges après fissuration, où un maximum de $242 \% \mathrm{a}$ été atteint.

\subsection{Ductilité}

Dans les bétons de fibres, c'est la ductilité qui est visée pour une rentabilité du matériau. Après fissuration du matériau, des charges importantes peuvent être reprises, même sous des déformations relativement importantes.

Les indices de ductilité calculés à partir des courbes charges-flèches d'après la norme ASTM 1018 sont présentés dans la figure 5 . Ces indices témoignent séparément l'apport de la matrice, du dosage en fibres et de l'élancement. On constate qu'il y a peu de différence au niveau dans la zone pré pic ; par contre dans la phase post-pic, la différence est remarquable. Pour cette raison, les indices de ductilité $\mathrm{I}_{5}, \mathrm{I}_{10}$ et $\mathrm{I}_{20}$ sont relativement peu sensibles et ne permettent pas de différencier le comportement en flexion des spécimens. Nous nous limitons au calcul des indices de ductilité $\mathrm{I}_{30}, \mathrm{I}_{50}$ et $\mathrm{I}_{100}$.

En se basant sur la figure 5, les observations suivantes peuvent être faites :

- Une augmentation du dosage en fibres améliore la ductilité et la capacité d'absorption d'énergie.

- Un dosage élevé résulte en une grande capacité de reprise de charge plus élevée à de grandes flèches.

- Les indices de ductilité sont faibles pour un béton à haute résistance comparé aux bétons ordinaires et autoplaçants. L'effet de l'orientation des fibres est dominant.

Il faut noter que le fait de doubler le dosage de 0,5 à $1 \%$ n'entraîne pas le dédoublement de la ductilité, une différence de $30 \%$ est observée pour l'indice $\mathrm{I}_{100}$. L'élancement des fibres a une faible influence sur la ductilité pour tous les indices de ductilité pour un dosage variant de 0,5 à $1 \%$.

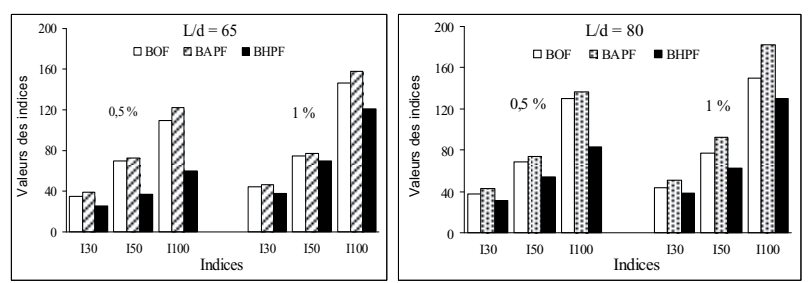

Fig. 5. Indices de ductilité

\subsection{Comportement cyclique des bétons}

\subsubsection{Analyse globale}

La figure 6 représente l'énergie consommée et cumulée en fonction de la flèche. Cette énergie est mesurée comme étant l'aire sous la courbe charge-flèche aux déplacements $1,2,3,4$ et $5 \mathrm{~mm}$. Cette énergie reste supérieure pour les BAPF par rapport aux BHPF et BOF. On peut également constater que pour les petites flèches, toutes les configurations se rapprochent pour se disperser aux grandes flèches. Ainsi l'énergie dissipée par $1 \%$ de fibres est plus importante que celle dissipée par $0,5 \%$ de fibres et ceci pour les deux élancements.

Dans le domaine post-pic, les crochets entraînent une dissipation d'énergie importante par leur glissement. La rupture de la fibre doit d'être évitée pour favoriser la rigidité du matériau. Toutes les courbes montrent que les fibres sont inactives au début du chargement et rentrent en action après fléchissement des spécimens. 


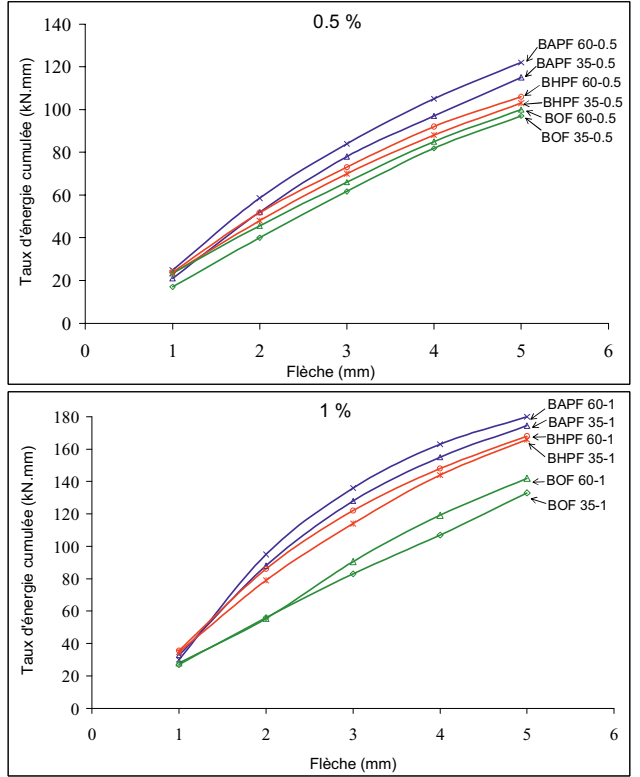

Fig. 6. Taux d'énergie cumulée en fonction de la flèche

\subsubsection{Analyse locale}

A partir des courbes expérimentales charge-flèche qui comprennent plusieurs boucles charges-décharges, une analyse locale est faite afin d'étudier l'influence de l'ancrage sur la réversibilité du comportement. Dans un matériau, il est difficile de séparer les phénomènes propres aux déformations de ceux liés à la rupture, car les microfissures existent déjà avant toute sollicitation. $\mathrm{Au}$ dessous d'une certaine valeur de sollicitation, les déformations sont dues aux mouvements réversibles.

L'irréversibilité de ces mouvements permet de définir conventionnellement la limite élastique. La rupture par décohésion des liaisons pâte-granulats constitue les premières déformations irréversibles; les microfissures au niveau des liaisons commencent à progresser conduisant ainsi au niveau macroscopique à des déformations permanentes qui s'ajoutent aux déformations élastiques. Pour des sollicitations plus élevées, les microfissures se propagent en s'alignant dans la direction des contraintes de traction.

Afin de quantifier ces différences de déplacements, nous allons recourir à la notion de degré de réversibilité (R) que l'on définit par le rapport entre le déplacement réversible $\left(D_{r}\right)$ et le déplacement total $\left(D_{t}\right)$ [5]. Le déplacement réversible est la distance entre l'axe des abscisses du grand axe de la boucle décharge-charge et flèche au moment de la décharge, figure 7.

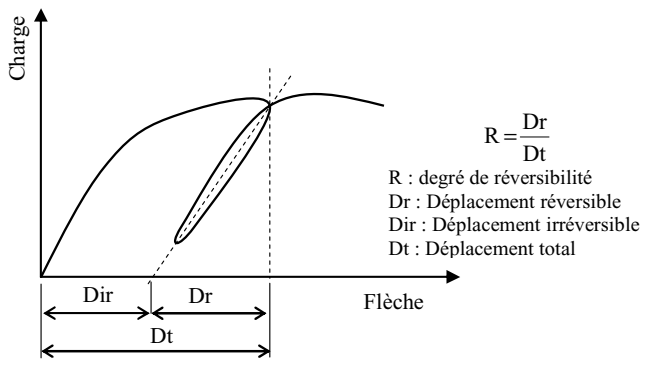

Fig. 7. Définition du degré de réversibilité
Le déplacement réversible est obtenu par la distance entre l'axe des abscisses du grand axe de la boucle décharge-charge et la flèche au moment de la décharge. La figure 8 montre que le degré de réversibilité est plus élevé pour des dosages de $1 \%$. L'élancement a aussi une influence importante; plus l'élancement est grand plus le degré de réversibilité est important.

L'analyse locale effectuée au niveau des ancrages permet d'étudier leur rigidité, leur influence sur la réversibilité du comportement et les conséquences sur le comportement vis-à-vis des sollicitations cycliques. L'ancrage de la fibre à crochets se redresse dans la masse de la matrice, ce qui contribue à améliorer la rigidité. Sur les sections de rupture, la quasi-totalité des crochets ont été redressés, signe d'une dissipation optimale d'énergie. Lors du déchargement, les ancrages ne retrouvent pas leur position initiale et leurs ruptures rendent le matériau moins rigide; traduit par des déplacements réversibles importants. Les résultats obtenus montrent que le fait d'avoir un ancrage très rigide rend le matériau plus souple, suite au décollement des crochets.
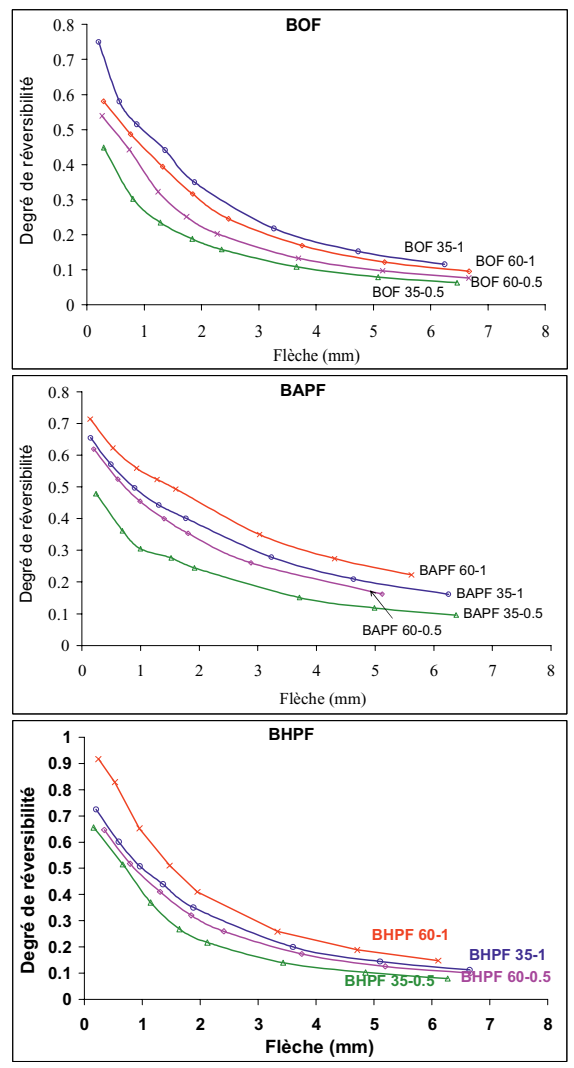

Fig. 8. Degré de réversibilité des différentes configurations

\subsection{Processus de fissuration des éprouvettes}

La figure 9 montre les différents états de fissuration à la rupture de spécimens fibrés et non fibrés, obtenues par la technique d'analyse d'image de la caméra numérique.

Pour les bétons non fibrés; l'éprouvette se rompt avec une seule fissure initiée au niveau de la fibre inférieure. La fissure se propage verticalement vers le haut, divisant le spécimen en deux parties (figure 9(a)). Pour les bétons fibrés et sous l'effet d'une redistribution de contraintes de 
traction dans la zone de flexion, la fissure principale est accompagnée par d'autres fissures indépendantes d'ouvertures assez importantes ou de petites fissures secondaires issues de la fissure principale. L'énergie de rupture est distribuée dans un grand volume de matériaux augmentant la largeur de la zone de flexion. Cela conduit à des charges maximales et une ductilité plus élevées pour les bétons fibrés. Il reste que l'orientation est le paramètre indispensable pour activer le transfert de charge de la matrice vers les fibres et de fibres en fibres. Ce transfert de charge est à l'origine de la multifissuration observée dans les cas des BOF et BAPF du fait de leur rhéologie améliorée (figures 9(b), (c)).
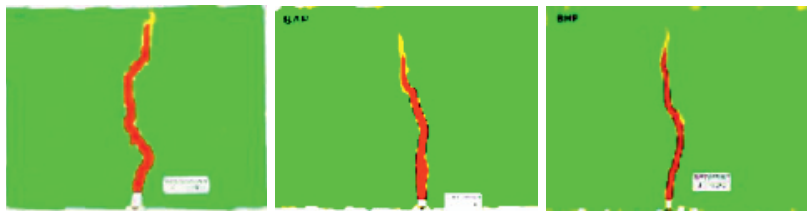

(a) Bétons non fibrés
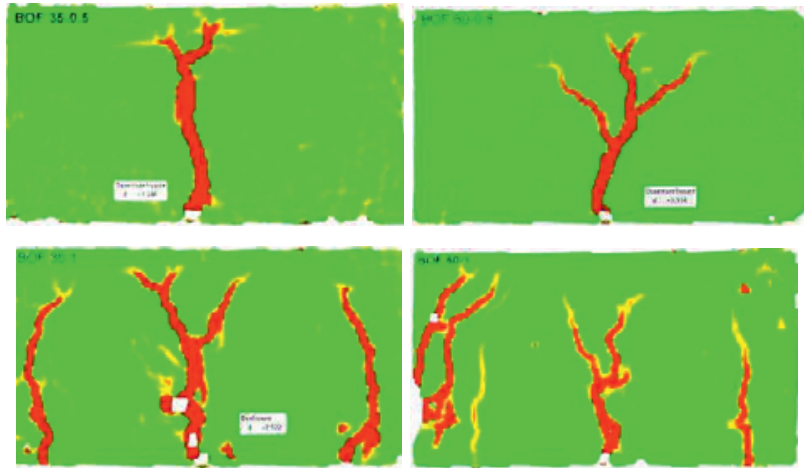

(b) Bétons ordinaires fibrés
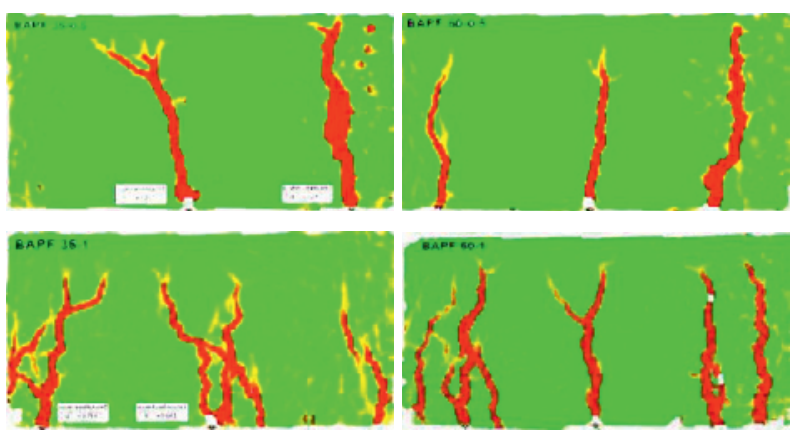

(c) Bétons autoplaçants fibrés
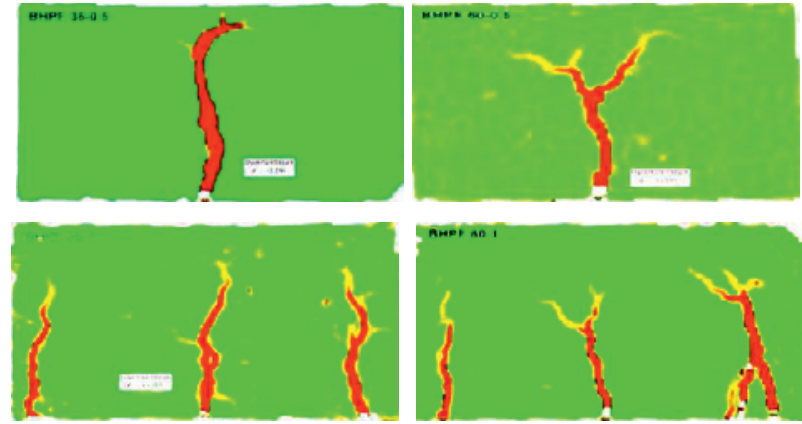

(d) Béton à hautes performances fibrés

Fig. 9. Processus de fissuration des spécimens

\section{Conclusions}

A partir des résultats des essais, on peut tirer les conclusions suivantes :

- Une augmentation quasi linéaire de la résistance à la flexion du béton fibré en fonction du dosage volumique des fibres.

- L'effet de la rhéologie est fondamental. Dans les bétons fluides, la répartition des fibres est homogène et les fibres sont orientées dans le sens de l'effort et par conséquent la ductilité des bétons fibrés est nettement améliorée.

- Les bétons autoplaçants ont montré la meilleure performance dans la reprise de charges après fissuration, où un maximum de $242 \%$ a été atteint.

- L'orientation est le paramètre fondamental pour l'activation du transfert de charge matrice-fibres ou fibres-fibres.

\section{References}

1. P. Rossi, Steel fiber reinforced concretes (SFRC): An example of french research, ACI Materials Journal, vol. 91, n³, (1994), pp. 273-279.

2. B. Boulekbache, M. Hamrat, M. Chemrouk, S. Amziane, Flowability of fibre-reinforced concrete and its effect on the mechanical properties of the material. Construction and Building Materials, vol. 24, n 9, (2010), pp. 1664-1671.

3. P. Soroushian, C.D. Lee, Distribution and orientation of fibers in steel fiber reinforced concrete, ACI Materials Journal, vol. 87, $\mathrm{n}^{\circ}$ 5, (1990), p. 433-439.

4. P. Stähli, R. Custer, J.G.M. Van Mier, On flow properties, fibre distribution, fibre orientation and flexural behaviour of FRC, Materials and Structures, vol. 41, n 1, (2008), p.189-196.

5. A. Bascoul, Etude de la contribution des fibres métalliques à l'amélioration du comportement du béton au cisaillement, Thèse de doctorat, Université de Toulouse, (1974). 